\title{
Preface
}

\section{From Bench to Bedside and Vice Versa}

$\mathrm{T}$

wo streams of my life as a neurologist joined to form this book: research in the laboratory and caring for patients in the hospital. It began when, as a young resident in neurology, my ambition was to become a good neuropsychiatrist. To achieve that mission, I knew I had to go beyond what is covered in textbooks, because they present what is known, and I wanted to prepare myself to anticipate the future. I saw that we needed to stop doing medicine in what I call the "Molière way," the manner characterized by the playwright in which doctors are long on discussion but short on substance. I thought that the best way to train good neurologists was to provide the students with a high level of teaching, which entails doing excellent research that would also benefit patients - a way of thinking not in fashion at that time. 
After a lengthy search, I was fortunate to find an excellent laboratory at the Collège de France in Paris, and for the next four years I devoted myself to neurobiochemistry research. When I returned to the clinic at the Salpêtrière Hospital after the years in the laboratory, I brought with me something unique at the time: double training as both a clinical neurologist and a neuroscience researcher. I never left the lab as I continued to see patients. Concurrently with clinical work, my research in my lab for over thirty-five years focused essentially on the physiological role of the basal ganglia, the structures deep within the cerebral hemisphere, the "dark basement in the brain," as described by Kinnier Wilson, that play a major role in processing the automatic motor behavior that I was observing in the examining room and, as detailed in the following pages, form part of the concept of subconsciousness.

My intention has been not to concentrate on the most understood mental cognitive functions such as memory, language, and consciousness, but instead to reinforce the neglected concept of automatic behaviors that had been studied in the past by psychologists (e.g., Pierre Janet and William James) or philosophers (e.g., Friedrich Nietzsche) under the name "subconscious," not to be confused with "unconscious" in the Freudian sense. My aim continues to be to explain what the subconscious is, its overlooked role in controlling 99 percent of our behavior, to trace its recently discovered anatomo-physiological substratum in the brain, with a special reference to one of the more ancient parts of 
the brain, the basal ganglia, and to examine what happens when it is ill.

The most famous disease resulting from damage to the basal ganglia is Parkinson's disease and in problems with controlling movements and posture. Patients' efforts to compensate for their loss of automatic behavior is a constant exhausting struggle. As Jean-Martin Charcot explains, "The Parkinsonian patient is condemned to produce voluntary movements for life." This prompted me to become primarily a movement disorder physician along with being a neuropsychologist.

However, research indicates that the role of the basal ganglia is not limited to movements. What we showed in my lab was that the basal ganglia also serve as the "hub" to process intellectual and emotional functions, which are all part of the concept of subconsciousness in normal persons. Further, we tried to answer the following questions: What about the concept of subconsciousness in humans compared with other animals? What distinguishes the faculty of subconsciousness from the classic concepts of consciousness and metaconsciousness? What happens when brain structures, in particular the basal ganglia, are dysfunctional, that is, when the processing of subconsciousness is abnormal?

In the examining room, I am always listening to and looking at patients very carefully. Here they are not masked by their social faces. They are totally natural and revealed as they truly are. They are giving subtle clues to their disorders, since I believe movement is the synthesis of our emotions, intellect, and physical condition. 
Bench and bedside continue to merge for me: science on the one hand and observing human behavior on the other; and this extends even to ordinary life. If I look down from my window at people walking by, as part of my own subconscious, I have a feeling for who they are from their posture, gait, and body movements. Today, for example, I saw a man with a particular gait and swinging of his arm, which raised a question in my mind as to whether he might develop a serious disorder in the future. What could we learn now about the basal ganglia that could alter the course of his disease?

As we continue to understand the subconscious, it becomes clear that it is time to reconsider the basal ganglia as a therapeutic target, not just for the improvement of motor disorders but also psychiatric disorders, if, as I propose, we are to find new and more effective ways to address these diseases. 